\title{
Die Messung des totalen Wirkungsquerschnittes von festem Neon und Argon mit sehr langsamen Neutronen
}

\author{
Von Tasso Springer \\ Labor für Technische Physik der Technischen Hochschule München \\ und Werner Wiedemann
}

\begin{abstract}
Kommission für Tieftemperaturforschung der Bayer. Akademie der Wissenschaften, München (Z. Naturforschg. 15 a, 828-830 [1960]; eingegangen am 6. Juli 1960)
\end{abstract}

\begin{abstract}
Der totale Wirkungsquerschnitt von festem Neon bei $20^{\circ} \mathrm{K}$ und von festem Argon bei $77^{\circ} \mathrm{K}$ und $20^{\circ} \mathrm{K}$ wurde mit monoenergetischen Neutronen zwischen $3 \cdot 10^{-4}$ und $2,6 \cdot 10^{-3} \mathrm{eV}$ im Transmissionsversuch gemessen. Unter Berücksichtigung des Umstandes, daß die theoretisch abgeschätzte unelastische Streuung nur einen kleinen Beitrag zum totalen Wirkungsquerschnitt liefert, erhält man hieraus den Absorptionsquerschnitt. Extrapolation desselben nach dem $1 / v$-Gesetz auf Neutronen von $v=2200 \mathrm{~m} / \mathrm{s}$ Geschwindigkeit $(0,025 \mathrm{eV})$ ergibt für Neon $\sigma_{\mathrm{a}}(2200 \mathrm{~m} / \mathrm{s}) \simeq 0,038 \pm 0,007$ barn. Im Hinblick auf die Unsicherheit in der Kenntnis des Streubeitrages soll dieser Wert mit den angegebenen Fehlergrenzen als eine obere Grenze angesehen werden. Ferner ergibt sich für die inkohärente Streuung am Neon eine obere Grenze $\sigma_{\text {incoh }}<0,1$ barn. Für Argon ergab sich $\sigma_{\mathrm{a}}=0,61 \pm 0,03$ barn in Übereinstimmung mit anderen Angaben.
\end{abstract}

Transmissionsversuche mit Neutronen ergeben den totalen Wirkungsquerschnitt $\sigma_{\mathrm{t}}$ des Probematerials, welcher gleich der Summe von Absorptionsquerschnitt $\sigma_{\mathrm{a}}$ und Streuquerschnitt $\sigma_{\mathrm{s}}$ ist. Für den Fall, daß die Neutronenenergie und die Probentemperatur sehr niedrig sind, ist der Beitrag der Streuung in vielen Fällen aus folgenden Gründen sehr klein: (a) Wenn die Probe ein Festkörper und die Neutronenwellenlänge größer als die BragGGrenzwellenlänge ist, so verschwindet die kohärente elastische Streuung. (b) Die unelastische Streuung ist gering, weil die Gitterschwingungen nicht merklich thermisch angeregt sind und weil sie nicht sehr durch das Neutron selbst angeregt werden können. Der einzige Streubeitrag kommt gegebenenfalls von der inkohärenten elastischen Streuung (Inkohärenz durch Isotopenmischung, Spin oder Gitterfehler); er ist in den meisten Fällen gering. Unter diesen Umständen wird $\sigma_{\mathrm{t}}$ praktisch gleich $\sigma_{\mathrm{a}}$; zumindest aber gibt $\sigma_{\mathrm{t}}$ eine obere Grenze für $\sigma_{\mathrm{a}}$. Vorteilhaft ist dabei, da $\beta \sigma_{\mathrm{a}}$ bei kleinen Neutronenenergien wegen des $1 / v$-Gesetzes sehr groß ist.

Der totale Wirkungsquerschnitt von festem Neon und Argon wurde in diesem Sinne in einem Energiebereich zwischen $3 \cdot 10^{-4}$ und $2,6 \cdot 10^{-3} \mathrm{eV}$ untersucht. Der Absorptionswirkungsquerschnitt von Neon ist von gewissem Interesse für die Reaktortechnologie.

$1 \mathrm{P}$. Höнne, wird veröffentlicht.

\section{Messung und Ergebnisse}

Die Neutronen wurden durch den mechanischen Schraubenselektor am FRM-Reaktor monochromatisiert $^{1}$. Seine Energieauflösung beträgt etwa $25 \%$. Infolge des starken Abfalls der Neutronenintensität mit abnehmender Energie innerhalb des Auflösungsintervalles (Abfall des Pilespektrums; Absorption in den Meßküvettenwänden) liegt der Schwerpunkt der Neutronenenergie merklich höher als die nominelle Energie, die man aus den mechanischen Daten des Selektors berechnen kann. Die „effektive“ Energie wurde deshalb mit Hilfe von Gold-Transmissionsmessungen experimentell bestimmt.

Unsere experimentelle Anordnung ist in Abb. 1 dargestellt. Als Probengefäß diente eine Kupferküvette (K), welche an den Boden des Wasserstoff-Vorratsgefäßes eines Kryostaten gelötet war. Es wurden zwei Küvetten (61 bzw. $100 \mathrm{~mm}$ lang, $2 \mathrm{~mm}$ Wandstärke) verwendet. Nach dem Abkühlen der vorher evakuierten Küvette auf $20^{\circ} \mathrm{K}$ wurde das Neon (reinst, Fa. Linde) vorsichtig eingefüllt. Der Druck im Puffergefäß lag während des Einfriervorgangs zwischen 5 und 1 atü. Das Einfrieren des größten Teiles des Gases dauerte ca. 20 Minuten. Die Wiederverdampfung erfolgte innerhalb weniger Minuten, indem gasförmiger Wasserstoff in den wärmeisolierenden Vakuumraum des Kryostaten eingelassen wurde. Die Neutronenintensität hinter der Küvette wurde mit einem Bohrzählrohr $B_{1}$, die einfallende Intensität mit einem als Monitor dienenden Zählrohr $\mathrm{B}_{2}$ gemessen.

Die Zählrate relativ zur Zählrate des Monitors, abhängig von der Neutronenenergie, zeigt Abb. 2, 
und zwar für die gekühlte $\left(20^{\circ} \mathrm{K}\right)$ und evakuierte Küvette $\left(I_{0}\right)$ und für die mit festem Neon bei $20^{\circ} \mathrm{K}$ gefüllte Küvette $(I)$. Der totale Wirkungsquerschnitt ist dann $\sigma_{\mathrm{t}}=(\mathrm{l} / n) \ln \left(I_{0} / I\right)$. Dabei enthält die vom Neutronenstrahl durchsetzte Schicht $n$ Atome pro $\mathrm{cm}^{2}$ des Strahlquerschnittes. $n$ läßt sich aus der Probendichte $\varrho$, der Loschmidsschen Zahl und dem Atomgewicht berechnen. Die Dichte $\varrho$ des festen Neons ergab sich aus der eingefüllten Gasmenge zu $1,10 \pm 0,04 \mathrm{~g} / \mathrm{cm}^{3}$. (In der Literatur werden die

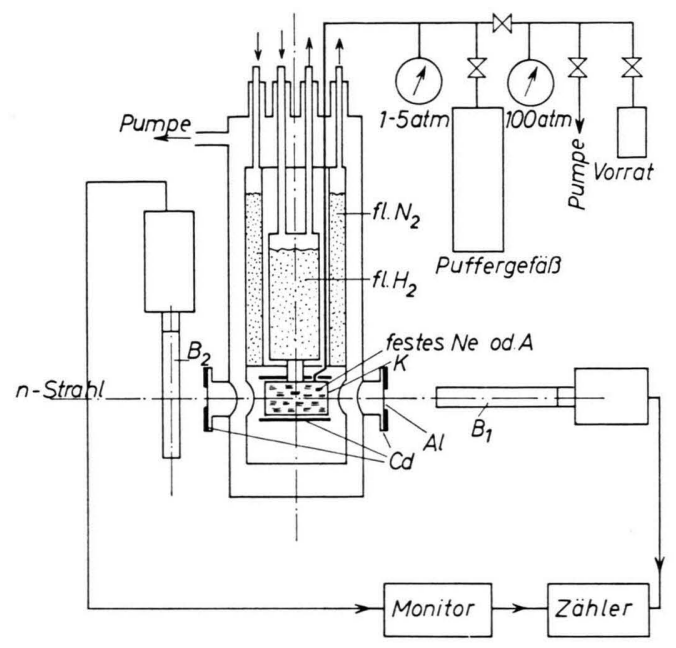

Abb. 1. Kryostat für Transmissionsversuche.

Werte $1,0 \mathrm{~g} / \mathrm{cm}^{3}$ [s. Anm. ${ }^{2}$ ] bzw. $1,20 \mathrm{~g} / \mathrm{cm}^{3}$ [s. Anm. ${ }^{3}$ ] angegeben. Der Wert 1,0 ist wahrscheinlich fehlerhaft, da er unter der Dichte des flüssigen Neons liegt.) Die Energieabhängigkeit von $\sigma_{t}$ ist in Abb. 3 in doppeltlogarithmischem Maßstab dargestellt. Innerhalb der Fehlergrenzen folgt $\sigma_{t}$ einem $1 / v$-Gesetz ( $v=$ Neutronengeschwindigkeit). Der Wirkungsquerschnitt bei $3 \cdot 10^{-4} \mathrm{eV}$ wurde aus Abb. 3, sowie aus den Ergebnissen von zwei weiteren Messungen entnommen; es ergaben sich die Werte: 0,35 barn, 0,45 barn (61-mm-Küvette) und 0,45 barn (100-mmKüvette) ; das Mittel ist

$$
\sigma_{\mathrm{t}}=0,42 \pm 0,06 \text { barn für } 3 \cdot 10^{-4} \mathrm{eV} \text {. }
$$

Die starke Zunahme von $\sigma_{t}$ bei Energien oberhalb $2 \cdot 10^{-3} \mathrm{eV}$ kommt von unelastischen Streuprozessen, bei denen das Neutron Energie an das Kristallgitter abgibt. Bei dem Meßpunkt mit der höchsten verwendeten Energie rührt sie ferner von der Annäherung des Monochromator-Auflösungsbereiches an die BRAGG-Grenze her. Unelastische Streuvorgänge, bei denen das Neutron Energie vom Gitter gewinnt, spielen nur eine geringe Rolle. Eine quantitative Abschät- zung der Anteile der unelastischen Streuung wurde mit den Formeln von Kothari und Singwi ${ }^{4}$ durchgeführt. Die Ergebnisse bei $20^{\circ} \mathrm{K}$ zeigt Tab. 1.

Zieht man von dem oben angegebenen Wert von $\sigma_{\mathrm{t}}$ den abgeschätzten unelastischen Streuquerschnitt von 0,07 barn ab (Tab. 1), und nimmt man an, der Rest

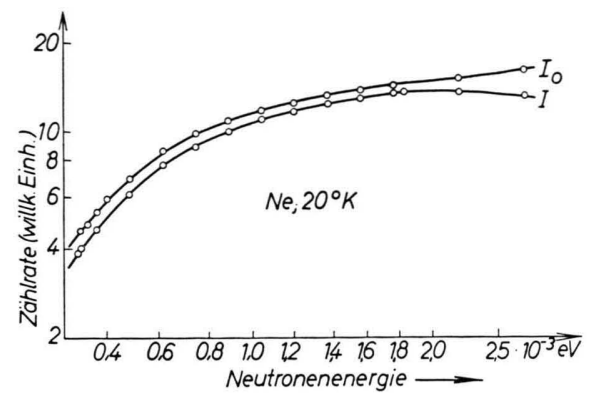

Abb. 2. Neutronienintensität bei leerer Küvette $\left(I_{0}\right)$ und bei gefüllter Küvette $(I)$.

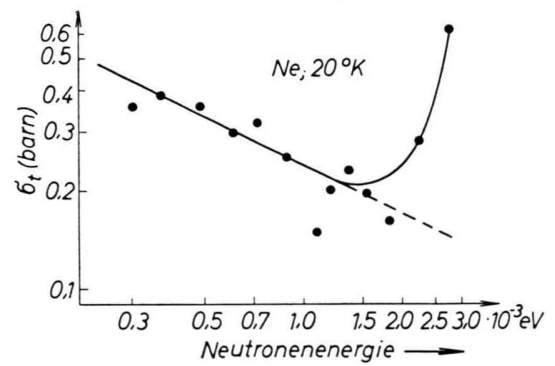

Abb. 3. Totaler Wirkungsquerschnitt des Neons.

sei ausschließlich der Absorption zuzuschreiben, so erhält man, wenn man nach einem $1 / v$-Gesetz auf $0,025 \mathrm{eV}(2200 \mathrm{~m} / \mathrm{s})$ extrapoliert

$\sigma_{\mathrm{a}}(2200 \mathrm{~m} / \mathrm{s})=[(0,42 \pm 0,06)-0,07]$

$\cdot\left(0,3 \cdot 10^{-3} / 25 \cdot 10^{-3}\right)^{1 / 2}=0,038 \pm 0,007$ barn.

Dieser Wert ist mit einer über die experimentellen Fehlergrenzen hinausgehenden Unsicherheit behaftet, die von der Unkenntnis des Beitrages der elastischen inkohärenten Streuung herrührt. Mit den angegebenen Fehlergrenzen kann er jedoch sicher als obere Grenze von $\sigma_{\mathrm{a}}$ angesehen werden. Sie liegt erheblich tiefer als die an anderer Stelle angegebene von 2,8 barn [s. Anm. ${ }^{6}$ ] bzw. 0,35 barn [s. Anm. ${ }^{7}$ ]. Ferner kann man aus der Messung eine obere Grenze für den inkohärent-elastischen Streuquerschnitt (auf

2 Handbook of Chemistry and Physics, Cleveland 1959.

3 American Institute of Physics Handbook, New York 1957.

${ }^{4}$ L. S. Kothari u. K. S. Singwi, Solid State Physics 8, 131 ff., Academic Press, New York 1959.

5 B. Bernardes, Phys. Rev. 112, 1534 [1958].

6 D. J. Hughes u. R. B. Schwartz, BNL-325 [1958].

7 D. G. Henshaw, Phys. Rev. 111, 1470 [1958]. 


\begin{tabular}{|c|c|c|c|c|}
\hline & \multicolumn{2}{|c|}{ Argon } & \multicolumn{2}{|c|}{ Neon } \\
\hline $\begin{array}{l}\text { DEBYE- } \\
\text { Temperatur } \\
\quad \theta ;(\mathrm{k} \theta) \\
\text { gebundener } \\
\text { Streu- } \\
\text { querschnitt } \\
\sigma_{\mathrm{b}} \text { (barn) }(\mathrm{s}) \\
(\mathrm{s} . \text { Anm. } \\
\text { gemessene } \\
\text { gichte } \\
\varrho \quad\left(\mathrm{gcm}^{-3}\right)\end{array}$ & $93^{\circ} \mathrm{K}(8$ & $\left.10^{-3} \mathrm{eV}\right)$ & $73^{\circ} \mathrm{K}(6,3$ & $\begin{array}{l}\left.10^{-3} \mathrm{eV}\right) \\
\left.{ }^{7}\right]\end{array}$ \\
\hline $\begin{array}{l}\text { Neutronen- } \\
\text { energie } \\
E(\mathrm{eV})\end{array}$ & $0,3 \cdot 10^{-3}$ & $2,7 \cdot 10^{-3}$ & $0,3 \cdot 10^{-3}$ & $2,7 \cdot 10^{-3}$ \\
\hline$\sigma_{\text {in }}^{\prime}($ barn $)$ & $6 \cdot 10^{-3}$ & $3,7 \cdot 10^{-3}$ & $7 \cdot 10^{-2}$ & $5 \cdot 10^{-2}$ \\
\hline$\sigma_{\text {in }}^{\prime \prime}($ barn $)$ & $2 \cdot 10^{-5}$ & $4,6 \cdot 10^{-3}$ & $3 \cdot 10^{-4}$ & $6 \cdot 10^{-2}$ \\
\hline $\begin{array}{l}\sigma_{\text {in }}^{\prime}+\sigma_{\text {in }}^{\prime \prime} \\
\text { (barn) }\end{array}$ & $0,6 \cdot 10^{-2}$ & $0,8 \cdot 10^{-2}$ & $7 \cdot 10^{-2}$ & $11 \cdot 10^{-2}$ \\
\hline $\begin{array}{l}\sigma_{\text {in }}^{\prime}+\sigma_{\text {in }}^{\prime \prime} \\
(\text { barn }) *\end{array}$ & $9 \cdot 10^{-2}$ & $8 \cdot 10^{-2}$ & - & - \\
\hline
\end{tabular}

Tab. 1. Unelastische Streuquerschnitte (berechnet nach ${ }^{4}$ ) und die verwendeten Daten von Argon und Neon. $\sigma^{\prime}$ in beschreibt inelastische Prozesse, bei denen das Neutron Energie von Kristallschwingungen gewinnt; diese Größe ist für sehr kleine Energien proportional $E^{-1 / 2}$, sie hat also denselben Verlauf wie $\sigma_{\mathrm{a}}$ und kann, wenn man die Temperatur nicht ändert, von $\sigma_{\mathrm{a}}$ experimentell nicht unterschieden werden. $\sigma^{\prime \prime}$ in beschreibt unelastische Prozesse, bei denen das Neutron Energie an den Kristall abgibt; $\sigma^{\prime \prime}$ in nimmt sehr stark mit der Neutronenenergie ab (etwa proportional $E^{3}$ ) und ist für die kleinen Energien völlig vernachlässigbar. Bezüglich des elastischen inkohärenten Streuanteils siehe Text. Alle Werte beziehen sich auf $20^{\circ} \mathrm{K}$, außer die der letzten Zeile ${ }^{*}$, die für $77^{\circ} \mathrm{K}$ gerechnet wurden.

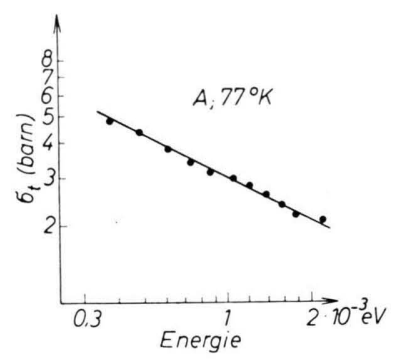

Abb. 4. Totaler Wirkungsquerschnitt des Argons.

Grund von Isotopeninkohärenz) entnehmen, und zwar $\sigma_{\text {incoh }}<0,1 \pm 0,1$ barn.

Es wurde weiter auch der totale Wirkungsquerschnitt von festem Argon* in der beschriebenen Weise ermittelt. Die Materialdichte wurde wieder aus der eingefüllten Gasmenge bestimmt. Bei allen durchgeführten Messungen lag diese zwischen 1,48 und $1,54 \mathrm{~g} / \mathrm{cm}^{3}$. Damit liegt die Dichte etwas unter

\footnotetext{
* Fa. L i n d e , vorgereinigt $(99,9 \%$ rein).
}

dem in der Literatur ${ }^{2}$ angegebenen Wert von 1,65 $\mathrm{g} / \mathrm{cm}^{3}$, was von der geringeren Kompaktheit des aus der Gasphase direkt ausgefrorenen Argons herrührt. Das Ergebnis einer der Messungen bei $77^{\circ} \mathrm{K}$ (flüssiger Stickstoff) zeigt Abb. 4 . $\sigma_{\mathrm{t}}$ folgt einem $1 / v$ Gesetz im Rahmen der Meßgenauigkeit. Der Beitrag der unelastischen Streuung spielt keine merkliche Rolle (siehe Tab. 1). Die isotopen- und spininkohärente elastische Streuung ist vernachlässigbar klein (gg-Kern ${ }_{18} \mathrm{~A}^{40}$ mit $99,6 \%$ ). Umrechnung auf die übliche thermische Energie von $0,025 \mathrm{eV}$ ergibt

$$
\sigma_{\mathrm{a}}(2200 \mathrm{~m} / \mathrm{s})=0,61 \pm 0,03 \text { barn. }
$$

\begin{tabular}{|c|c|c|}
\hline $\begin{array}{c}\sigma_{\mathrm{a}}(2200 \mathrm{~m} / \mathrm{s}) \\
(\text { barn })\end{array}$ & Methode & Literaturzitat \\
\hline $0,62 \pm 0,04$ & $\begin{array}{l}\text { Pile- } \\
\text { Oszillator }\end{array}$ & $\begin{array}{l}\text { F.Colmer u. D.J.Littler, } \\
\text { Proc. Phys. Soc., Lond. } \\
\text { A } 63,1175(1950) \text {. }\end{array}$ \\
\hline $0,60 \pm 0,10$ & $\begin{array}{l}\text { aus } \sigma_{\mathrm{t}}-\sigma_{\mathrm{s}} \\
\text { bei } 0,025 \mathrm{eV}\end{array}$ & $\begin{array}{l}\text { E. Melkonian u. a., Phys. } \\
\text { Rev. 73, } 1399 \text { (1948), und } \\
\text { S. Harris, Phys. Rev. 80, } \\
20 \text { (1950). }\end{array}$ \\
\hline $0,66 \pm 0,04$ & $\begin{array}{l}\text { Absorp- } \\
\text { tions- } \\
\text { messung }\end{array}$ & BNL -325 (s. Anm. ${ }^{6}$ ) \\
\hline$(0,53 \pm 0,03)$ & $\begin{array}{l}\mathrm{A}^{41}-110 \mathrm{~min}- \\
\text { Aktivie- } \\
\text { rungs- } \\
\text { querschnitt } \\
\text { allein }\end{array}$ & $\begin{array}{l}\text { S. Katcoff, Phys. Rev. } \\
\mathbf{8 7}, 886 \text { (1952). }\end{array}$ \\
\hline $0,61 \pm 0,03$ & $\begin{array}{l}\text { aus } \sigma_{\mathrm{t}} \text { mit } \\
\text { sehr } \\
\text { langsamen } \\
\text { Neutronen }\end{array}$ & diese Arbeit. \\
\hline
\end{tabular}

Tab. 2. Thermischer Absorptionsquerschnitt des Argons.

Der angegebene Fehler ist das quadratische Mittel folgender Fehler:

(a) herrührend von der Streuung der Meßpunkte in Abb. 4: $\pm 0,02$ barn;

(b) von dem Fehler in der effektiven Energie: $\pm 0,02$ barn und

(c) von der Unsicherheit in der Dichte: $\pm 0,012$ barn.

Eine weitere Messung bei $20{ }^{\circ} \mathrm{K}$, welche mit einer geringeren statistischen Genauigkeit durchgeführt worden war, ergab ebenfalls $0,60 \pm 0,04$ barn, woraus man erkennt, daß der Einfluß der unelastischen Streuung innerhalb der experimentellen Fehlergrenzen vernachlässigbar ist. Das Ergebnis wird in Tab. 2 mit den Daten anderer Messungen verglichen. Unser Wert stimmt mit den in der 1. und 2. Zeile angegebenen innerhalb der Fehlergrenzen überein. 